\title{
How we made the Li-ion rechargeable battery
}

\author{
Progress in portable and ubiquitous electronics would not be possible without rechargeable batteries. \\ John B. Goodenough recounts the history of the lithium-ion rechargeable battery.
}

\author{
John B. Goodenough
}

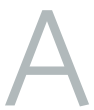

battery contains one or many identical cells. Each cell stores electric power as chemical energy in two electrodes, the anode and the cathode, which are separated by an electrolyte. The chemical reaction between the electrodes has an ionic and an electronic component. The electrolyte transports the ionic component inside a cell and forces the electronic component to traverse an external circuit. In a rechargeable battery, the chemical reaction is reversible.

In the 1960s, chemists in Europe were exploring the chemistry of reversible insertion of lithium into layered transition-metal sulfides. At that time, rechargeable batteries used strongly acidic $\left(\mathrm{H}_{2} \mathrm{SO}_{4}\right)$ or alkaline $(\mathrm{KOH})$ aqueous electrolytes that offered fast hydrogen-ion $\left(\mathrm{H}^{+}\right)$diffusion. The most stable cells used an alkaline electrolyte and a layered nickel oxyhydroxide $(\mathrm{NiOOH})$ as the cathode into which $\mathrm{H}^{+}$is inserted reversibly to form the hydroxide $\mathrm{Ni}(\mathrm{OH})_{2}$. However, an aqueous electrolyte limits the voltage of the cell and, therefore, the density of electric power a battery can deliver.

In 1967, Joseph Kummer and Neill Weber of the Ford Motor Company discovered fast sodium-ion diffusion above $300{ }^{\circ} \mathrm{C}$ in a ceramic electrolyte and invented a sodium-sulfur rechargeable battery that used the solid ceramic as the electrolyte, molten sodium as the anode and molten sulfur containing carbon felt as the cathode. The high operating temperature of above $300{ }^{\circ} \mathrm{C}$ made their battery commercially impractical, but it stimulated research into solid electrolytes and alternative battery strategies. At the time, I was working on transition-metal oxides at the MIT Lincoln Laboratory, and I was asked to monitor the development of this battery. The assignment introduced me to electrochemistry and to the challenge of developing a better oxide-based sodium-ion conductor. In response, I developed, together with Henry Hong, a $\mathrm{Na}_{1+x} \mathrm{Zr}_{2} \mathrm{Si}_{x} \mathrm{P}_{3-x} \mathrm{O}_{12}$ electrolyte that had a framework structure and supported fast sodium ion conductivity; it was dubbed NASICON (NA SuperIonic CONductor) by colleagues after I left for the University of Oxford in 1976.

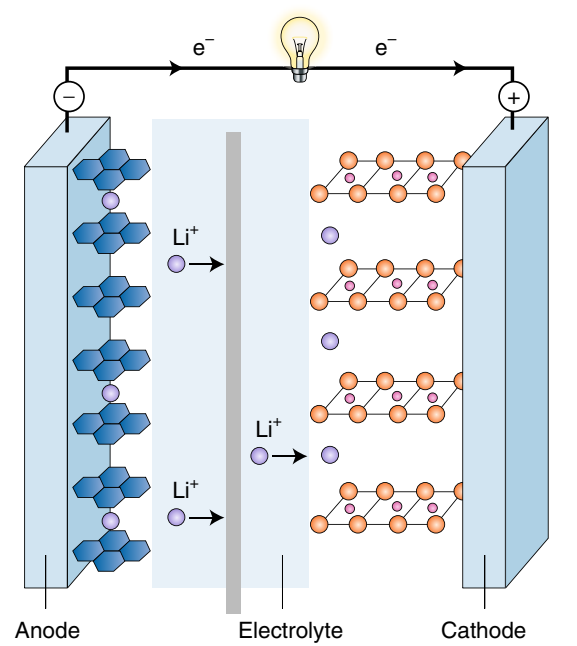

Fig. 1 | Components of a rechargeable Li-ion battery.

The oil crisis of the early 1970s exposed the vulnerability of US society, among others, to its dependence on imported oil and subsequently prompted investigations into solar and wind energy as potential sources for electric power. However, the intermittency of solar and wind meant that storage of this power would be required for these renewable sources to be useful. Hence, there was a desire for better rechargeable batteries.

Non-rechargeable batteries using a lithium anode and an organic-liquid electrolyte were known at the time, so the next step was to use the chemistry demonstrated in Europe of reversible lithium-ion insertion into transition-metal layered sulfide cathodes in order to create a rechargeable battery. Stanley Whittingham was hired by the Exxon Mobil Corporation to create such a rechargeable battery with a layered titanium disulfide $\left(\mathrm{TiS}_{2}\right)$ cathode that discharged to $\mathrm{LiTiS}_{2}$ through reversible lithium insertion. In 1976, Whittingham reported good initial performance for the cell. However, on repeated charging, plating of the lithium anode resulted in the formation of anode dendrites (lithium whiskers) that grew across the electrolyte to the cathode to create an internal short circuit, which would ignite the flammable electrolyte. As a result, the program was abandoned a few years later.

Around this time, my group at the University of Oxford was investigating how much lithium can be extracted from a layered lithium cobalt oxide $\left(\mathrm{LiCoO}_{2}\right)$ or lithium nickel oxide $\left(\mathrm{LiNiO}_{2}\right)$ before the structure changed. I had reasoned that a rechargeable battery could be fabricated in a discharged, as well as a charged, state. We were able to demonstrate that over half of the lithium could be extracted reversibly with these cathode materials. This led Akira Yoshino, then at the Asahi Kasei Corporation, to make the first lithium-ion rechargeable battery by combining the $\mathrm{LiCoO}_{2}$ cathode with a graphitic-carbon anode (Fig. 1). This battery was used by the Sony Corporation to power the very first portable phone.

To lower the cost and increase safety, an all-solid-state rechargeable battery - where the liquid electrolyte is replaced with a solid electrolyte - is an active future direction for battery research. Indeed, rechargeable batteries with solid electrolytes are now being developed, including sodium batteries with a NASICON electrolyte. In 2015, a remarkable dielectric amorphousoxide solid electrolyte was brought to me by Maria Helena Braga of the University of Porto. It has lithium and sodium ion conductivities comparable to those of the organic-liquid electrolytes used in today's lithium-ion batteries. At the University of Texas at Austin, where I moved to in 1986, we have used this unique solid to develop all-solid-state rechargeable batteries that are able to plate dendrite-free alkali-metal anodes with good contact over a long cycle life at acceptable charge and discharge rates. These developments suggest that all-solidstate batteries may soon be used to power all-electric road vehicles that are competitive with vehicles powered by fossil fuel in an internal combustion engine.

\section{John B. Goodenough \\ University of Texas at Austin, Austin, TX, USA. e-mail: jgoodenough@mail.utexas.edu}

Published online: 9 March 2018 https://doi.org/10.1038/s41928-018-0048-6 